\title{
EVALUATING OF TWO TYPE OF CYCLOSPORINE-A CONTAINING NANOSUSPENSION FOR OPHTHALMIC ADMINISTRATION
}

\author{
Tughan Duran ${ }^{1}$, Osman Karakus ${ }^{2}$, Tuncer Degim ${ }^{3}$, Burcu Eser ${ }^{4}$, Sermet \\ Sezigen ${ }^{5}$, Zafer Guney ${ }^{2}$, Canan Uluoglu ${ }^{2}$ \\ ${ }^{1}$ Department of Refractive Surgery, Maya Eye Hospital, Ankara, Turkey \\ ${ }^{2}$ Department of Pharmacology, Gazi University Faculty of Medicine, Ankara, Turkey \\ ${ }^{3}$ Department of Pharmaceutical Technology, Biruni University Faculty of Pharmacy, Istanbul, Turkey \\ ${ }^{4}$ Institute of Health Sciences, University of Health Sciences, Ankara, Turkey \\ ${ }^{5}$ Department of Medical CBRN Defense, University of Health Sciences, Ankara, Turkey
}

Address for Correspondence: Canan Uluoglu. E-mail: culuoglu@yahoo.com

Received: 11.05.2021; Accepted: 06.08.2021; Available Online Date: 20.09.2021

(c) Copyright 2021 by Dokuz Eylül University, Institute of Health Sciences - Available online at https://dergipark.org.tr/en/pub/jbachs

Cite this article as: Duran T, Karakus O, Degim T, Eser B, Sezigen S, Guney Z, Uluoglu C. Evaluating of two type of cyclosporine-a containing nanosuspension for ophthalmic administration. J Basic Clin Health Sci 2021; 3: 107- 116.

\begin{abstract}
Dry eye is both a common symptom and a disease. It is known that the ophthalmic emulsion of the immunomodulator cyclosporine-A (Сyc-A) has a positive effect on this condition but its' absorption to intraocular tissues is limited. Nanosuspension is a drug formulation that aims to increase the bioavailability. The aim of this study was to develop a Cyc-A nanosuspension formulation for a better intraocular absorption via ocular delivery and to investigate the effectiveness of the formulation by comparing it with two marketed ophthalmic emulsions (Restasis $\AA$ and Depores $\AA$ ). Two types of Cyc-A loaded Eudragit $\$ 100$ nanosuspension ( $\mathrm{A}$ and $\mathrm{B}$ ) were prepared. Drug formulations were applied to both eyes of 20 male Albino New Zealand rabbits with an interval of 12 hours for 14 days. In vitro drug release was tested using a dialysis sac and quantitative analysis was performed by HPLC for evaluating Cyc-A amounts in all formulations. Although all four formulations had similar particle size and polydispersity indexes, nanosuspension B which had a positive zeta potential value, had released more Cyc-A than other formulations. It was showed that Cyc-A loaded nanosuspension formulations which had good ocular tolerability could be a better alternative to commercial formulations for the treatment of dry eye. The nanosuspensions have ideal mean particle size range with a positive surface charge for ophthalmic applications of Cyc-A.
\end{abstract}

Keywords: Cyclosporine-A; Nanosuspension; Dry eye

\section{INTRODUCTION}

Dry eye, which is either the reduction of tear production or the excessive evaporation of the tear causes damage to the surface of the eye. Dry eye has a wide range of symptoms including mostly foreign body sensation, burning, redness, and pain (1-9). Dry eye is a very common eye disease, and it affects 
about 4.3 million people over the age of 50 In the United States with a prevalence of $7 \%$ in females and $4 \%$ in males $(3,5,10-13)$.

Although the pathophysiology of dry eye syndrome (DES) is not fully understood; even today, it has only known that this syndrome is a part of the immunologically mediated inflammation affecting the lacrimal gland and ocular surface (14). It is estimated that the ophthalmic emulsion of Cyclosporine A (CycA), as an immunomodulatory agent, inhibits the synthesis and release of proinflammatory cytokines like TNF- $\alpha$ and IL-6. This inhibition positively affects the underlying inflammatory pathology which causes DES (12,14-16). extremely important in terms of efficacy of DES treatment.

The aim of this study was to develop new Cyc-A nanosuspension formulations for a better intraocular absorption via ocular delivery and to investigate the intraocular absorption of the formulations by comparing them with two marketed ophthalmic micro emulsions (Depores ${ }^{\mathrm{TM}}$ and Restasis ${ }^{\mathrm{TM}}$ which are accepted to be bioequivalent).

\section{METHODS \\ Materials}

Cyc-A ( $\geq 98.5 \%$ TLC), acetonitrile (HPLC grade), and methanol (HPLC grade) were purchased from Sigma-

Table 1. Concentration of Cyc-A in Depores, Restasis and Nanosuspension formulations.

\begin{tabular}{ccccc}
\hline Formulations & Depores & Restasis & Nanosuspension A & $\begin{array}{c}\text { Nanosuspension } \\
\text { B }\end{array}$ \\
\hline Cyc-A $(\mathrm{mg} / \mathrm{ml})$ & $0,50 \pm 0,2$ & $0,51 \pm 0,2$ & $0,52 \pm 0,3$ & $0,54 \pm 0,2$ \\
\hline
\end{tabular}

All values represented as mean values $(n=3, \pm$ Standard deviation (SD)0029)

The effect and the distribution of ophthalmic microemulsion forms of Cyc-A in ocular tissues have been investigated in various animal studies. Cyc-A accumulates the extraocular tissues more than intraocular tissues, especially in the cornea. However, the cornea acts as a barrier and it limits Cyc-A absorption. This limitation causes to the slow release of Cyc-A to the corneal epithelium and other tissues (17).

Nanosuspension is a pharmaceutical dosage form which is prepared by spreading drug particles in submicron sizes in the formulation. The aim of nanosuspension preparation is to increase the solubility of the drug by reducing the particle size and increasing the surface area of the drug particles in order to achieve higher drug absorption at the targeted tissues. Nanosuspension is not only improve the drug solubility, but also changes the pharmacokinetics of the drug. It also increases drug safety and efficacy. Among the benefits of the nanosuspension form, the increased absorption of water-insoluble drugs, achieving the desired dose concentration at the targeted tissue and having high physical/chemical stability can be considered (18-20). Although Cyc-A has being used for a long time, there are still several studies which focus on increasing the concentration of the drug in the eye. In this regard, determination of intraocular absorption of Cyc-A is
Aldrich. Depores $0.05 \%$ microemulsion (Deva Holding Inc.) and Restasis $0.05 \%$ microemulsion (Allergan) were purchased commercially. Eudragit RS 100 was purchased from Röhm Pharma, GmbH, Darmstadt, Germany. All other chemicals were of analytical grade and commercially available.

\section{HPLC analysis}

Instruments and chromatographic conditions

A high-performance liquid chromatography (HPLC) system (Shimadzu, Kyoto, Japan), which was equipped with LC-20AD pump, SIL-20AC autosampler, Chromolith column (C-18, $150 \times 4.6$ $\mathrm{mm}, 5 \mu \mathrm{m}$ ), and SPD-M20A UV detector was used for the determination of absorbed Cyc-A concentration was used. The column temperature was $50^{\circ} \mathrm{C}$. The wavelength was set to $205 \mathrm{~nm}$. The mobile phase was a mixture of $200 \mathrm{ml} \% 0,1$ trifluoroacetic acid (TFA) containing water and $800 \mathrm{ml}$ acetonitrile. The flow rate was $1.0 \mathrm{~mL} / \mathrm{min}$. The method was found to be linear $\left(r^{2}=0.998\right)$, reproducible, and sensitive (LOD $=0,001 \mathrm{microgram} / \mathrm{ml}, \mathrm{LOQ}=0.01 \mathrm{microgram} / \mathrm{ml}$ ).

\section{Standard solutions}

Stock solutions of Cyc-A were prepared in methanol. Series of standard standards were prepared in water. 


\section{Sample preparation}

250 microliters $(\mu \mathrm{l})$ sample of aqueous humor was added to $1 \mathrm{ml}$ of acetonitrile. After mixing 30 seconds, the mixture was centrifuged for $5 \mathrm{~min}$ at $10.000 \mathrm{rpm}$. Then $1 \mathrm{ml}$ of supernatant was taken and samples were dried under nitrogen stream. Then it was recovered with $200 \mu \mathrm{l}$ of a mixture of $65 \%$ acetonitrile, $15 \% \mathrm{H}_{2} \mathrm{O}$, and $20 \%$ ethanol. Finally, 10 $\mu \mathrm{l}$ of samples were injected into the HPLC. situ precipitation of the polymer and Cyc-A with the formation of matrix-type nanoparticles $(21,22)$. Then, the mixture was left under vacuum at $4^{\circ} \mathrm{C}$ for 24 hours. Finally, the mixture was filtered through a 0.2 micrometer membrane.

Preparation of nanosuspension B: The same preparation technique that was used nanosuspension

Table 2. Characterization of Depores, Restasis, and Nanosuspension B formulation.

\begin{tabular}{lccc}
\hline Formulations & Particle Size $(\mathrm{nm})$ & Polydispersity Index & Zeta Potential $(\mathrm{mV})$ \\
\hline Depores & 384,3 & $31,3 \%$ & $-26,3$ \\
Restasis & 203,4 & $25,1 \%$ & $-36,6$ \\
Nanosuspension B & 365,8 & $37,6 \%$ & 27,5 \\
\hline
\end{tabular}

\section{Preparation and the characterization of Cyc-A} nanosuspension formulations

Preparation of nanosuspension A: Cyc-A nanosuspension which contained Eudragit RS 100 was prepared by using a quasi-emulsion solvent evaporation technique (21). The drug (15 mg) and polymer $(240 \mathrm{mg})$ were dissolved at room temperature in ethanol $(2.85 \mathrm{ml})$. The solution was slowly injected with a syringe at $0.5 \mathrm{ml} / \mathrm{min}$ into $30 \mathrm{ml}$ water containing Tween $80(0.02 \%, w / v)$ and benzalkonium chloride (BAK) $(0.1 \%, w / v)$ and it kept at a low temperature in an iced-water bath. During the injection, the mixture was vigorously stirred by an Ultra-Turrax disperser at $25.000 \mathrm{rpm}$. The solution immediately turned into a pseudo-emulsion of the drug and polymer in the aqueous phase. The counterdiffusion of ethanol and water in the emulsion turned into micro and nano-droplets respectively. The gradual evaporation of the organic solvent causes in
A was also used without adding BAK for the preparation of nanosuspension $B$.

\section{Cyc-A contents of nanosuspension formulations} HPLC analysis was performed in order to measure Cyc-A concentrations in Restasis, Depores, nanosuspension $\mathrm{A}$, and nanosuspension $\mathrm{B}$.

\section{Analysis of particle size and zeta potentials of nanosuspensions \\ The mean particle size and zeta potential of the prepared nanosuspensions were measured by using Litesizer 500 (Anton Paar, Austria). Samples were subjected to the particle size analyses before the experiments and they were diluted 10-20 times with particle-free (filtered) distilled water. \\ The encapsulation efficiency (EE) of nanosuspension was indirectly measured. Nanosuspension was}

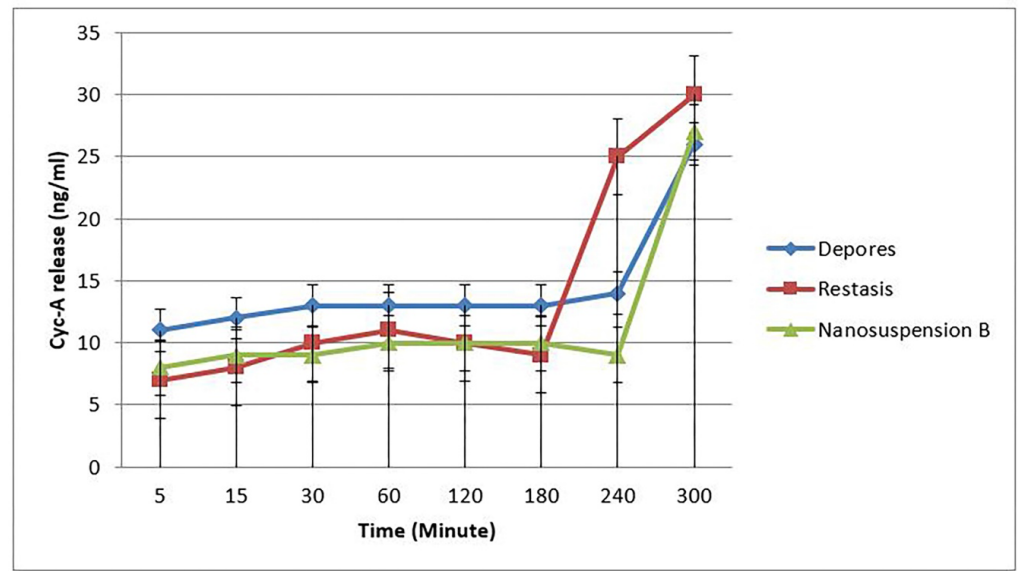

Figure 1. In vitro Cyc-A release profile of Depores, Restasis, and Nanosuspension B formulation as ng/ml (Error bars represent SEM, $\mathrm{n}=3$ ). 
centrifuged for 20 minutes at $10.000 \mathrm{rpm}$, then the supernatant was collected and analyzed by HPLC. The amount of encapsulated drug \% (EE\%) was obtained by dividing the total Cyc-A in the solution to the total Cyc-A in the supernatant.

\section{In vitro Cyc-A release studies}

In vitro Cyc-A release was determined by using a dynamic dialysis bag diffusion technique The dialysis sac (molecular weight cut-off $12.000 \mathrm{Da}$, Sigma) was filled with $0.5 \mathrm{~mL}$ of nanosuspension and it was immersed into $200 \mathrm{~mL}$ of saline at $37^{\circ} \mathrm{C}$. The solution was stirred with a magnet at $25 \mathrm{rpm} .1 \mathrm{~mL}$ aliquots were withdrawn and replenished with the fresh solution at predetermined time intervals. Finally, CycA amounts were determined by using HPLC.
B). A drop of drug formulation was applied to both eyes of rabbits with an interval of 12 hours for 14 days. At the end of 14 days, anesthesia was provided with Atropine $(0.1 \mathrm{mg} / \mathrm{kg}, \mathrm{SC})$, Xylazine $(5 \mathrm{mg} / \mathrm{kg}, \mathrm{IM})$, and Ketamine $(50 \mathrm{mg} / \mathrm{kg}, \mathrm{IM})$. Aqueous humor aspiration was performed from both chambers of the rabbits in a sterile condition. Then, Cyc-A concentrations were measured by using HPLC. The dose in the eye drop was determined by HPLC and the effective Cyc-A amount in the eye was calculated by diving the actual drug amount in the eye chamber with the dose in the applied formulation.

\section{Ocular irritation test}

Two male Albino New Zealand rabbit were used for ocular irritation test. This test was performed by using

Table 3. Cyc-A concentrations for Depores, Restasis, and Nanosuspension formulations after in vivo tissue (eye) penetration tests.

\begin{tabular}{|c|c|c|c|c|}
\hline Formulations & Depores & Restasis & Nanosuspension A & Nanosuspension B \\
\hline $\begin{array}{l}\text { Cyc-A concentration in } \\
\text { formulations }(\mathrm{mg} / \mathrm{ml})\end{array}$ & $0.50 \pm 0.2$ & $0.51 \pm 0.2$ & $0.52 \pm 0.3$ & $0.54 \pm 0.2$ \\
\hline $\begin{array}{l}\text { Cyc-A concentration in } \\
\text { aqueous humor }(\mathrm{mg} / \mathrm{ml})\end{array}$ & $0,12 \pm 0.6$ & $0.1 \pm 0.4$ & $0.02 \pm 0.01$ & $0.16 \pm 0.06$ \\
\hline $\begin{array}{l}\text { Released } \\
\text { Cyc-A\% at } 300 \text { minutes } \\
(\mathrm{mg})\end{array}$ & $25 \pm 0.4$ & $19,5 \pm 0.6$ & $4 \pm 0.04$ & $30.4 \pm 2.3$ \\
\hline
\end{tabular}

All values represented as mean values $(n=3, \pm$ Standard deviation (SD))

\section{In vivo animal study}

Male Albino New Zealand rabbits $(n=20)$ were included into the in vivo study which was approved by Gazi University Local Ethics Committee for Animal Experiments (G.U.ET-17.012) The whole study was conducted in accordance with the Basic \& Clinical Pharmacology \& Toxicology policy for experimental and clinical studies (23).

All rabbits $(2-3 \mathrm{~kg})$ were housed in an air-conditioned room $\left(22 \pm 2^{\circ} \mathrm{C}\right.$ and $55 \pm 5 \%$ relative humidity, and with a light/dark cycle of 12:12). They were allowed to access standard food and tap water ad libitum. The rabbits were randomly divided into four groups $(n=5)$ (Group 1: Depores, Group 2: Restasis, Group 3: Nanosuspension A, and Group 4: Nanosuspension only nanosuspension B as eye irritations such as swelling, discharge, and redness of the conjunctiva were observed during initial nanosuspension application. Nanosuspension B formulation was applied to the animals and their eyes were checked for two weeks with regard to ocular irritation. A drop of the drug was applied on both eyes of the rabbits with an interval of 12 hours for 14 days. Two rabbits were used as a control group.

\section{Statistics}

Drug release profiles were compared by using $\mathrm{F} 1$ and F2. F1 (Difference factor) and F2 (Similarity factor) were calculated for each formulation. $\mathrm{F}=0-15$ and 
$F 2=50-100$ values were accepted to be similar. F1 (1) and F2 (2) values were calculated as follows:

$f_{1}=\frac{\sum_{t=1}^{n}\left[R_{t}-T_{t}\right]}{\sum_{t=1}^{n} R_{t}} * 100$

$f_{2}=50 * \frac{100}{\sqrt{1+\frac{1}{n} \sum_{t=1}^{n}\left(R_{t}-T_{t}\right)^{2}}}$

All data were also compared with Kruskal Wallis and Mann-Whitney U post-hoc test. $(p<0.05)$.

\section{RESULTS}

Comparison of cyclosporine-A (Cyc-A) formulations

We prepared two different nanosuspension formulations of Cyc-A as nanosuspension $A$ and nanosuspension B. Result of HPLC analysis showed that nanosuspension $B$ contained much more amount of Cyc-A comparing Depores, Restasis, and nanosuspension A (Table 1). formulations. All values were reports as mean values of 12 measurements. SD values were less than 0.1 for all (Table 2).

\section{Drug release profiles}

Cyc-A is a hydrophobic substance and its water solubility is quite low. We decided to perform drug release studies in saline solution at $37^{\circ}$ in order to find potential formulation effect as we did not able to differentiate any formulation when we used an alcoholic dissolution medium. Although it was found that Restasis had higher in vitro Cyc-A release profile comparing other formulations, Nanosuspension B had a better percentage of in vitro Cyc-A release profile (Figure 1 and 2).

All F1 and F2 values were found to be out of the range $(0-15$ and $>50)$ except $F 2$ values for Depores and Restasis $(F 2=91.414)$. These results indicated that in vitro Cyc-A release profile of nanosuspension $B$ formulation was higher than Depores and Restasis as nanosuspension $B$ dissolved rather rapidly comparing other formulations.

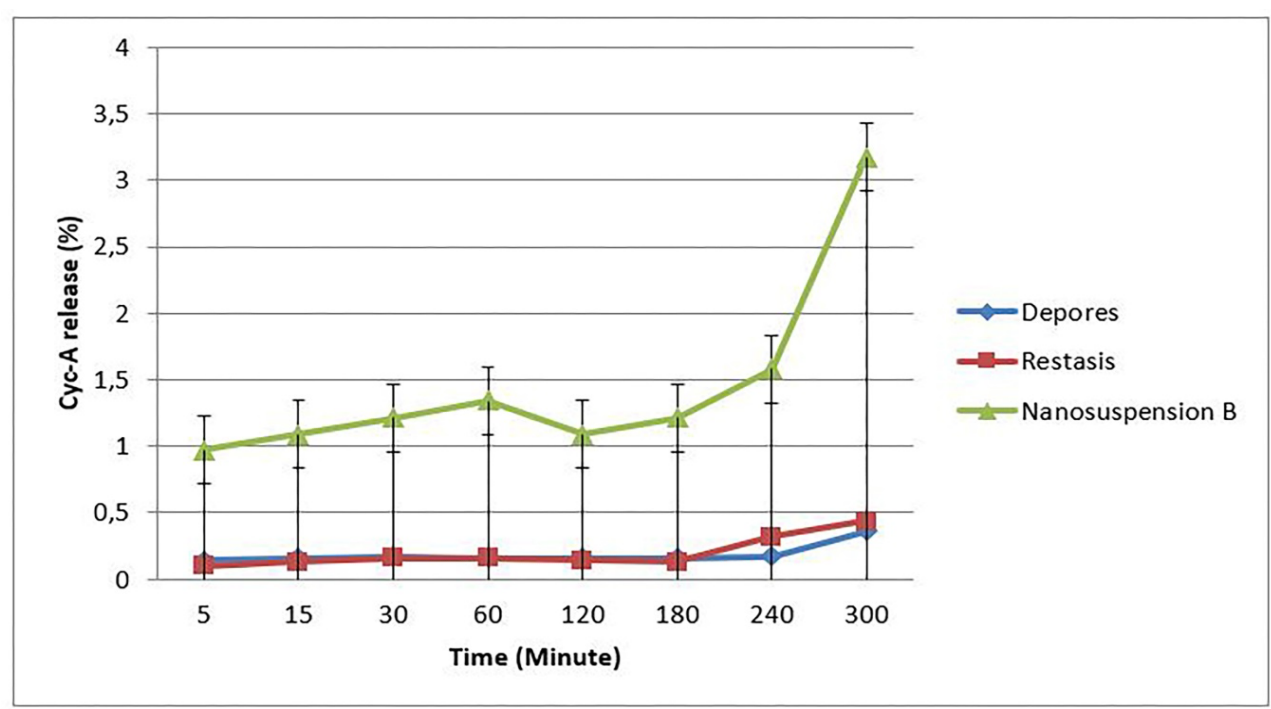

Figure 2. Percentage of in vitro Cyc-A release profiles for Depores, Restasis, and Nanosuspension B formulation (Error bars represent SEM, $n=3$ ).

Particle size analysis, zeta potentials and EE \% Although all formulations had similar particle size and polydispersity index, nanosuspension $B$ had a positive zeta potential values comparing with Depores and Restasis. The EE\% value was found to be higher than $95 \%$ for nanosuspension $B$. Lower values were obtained when BAK was present in the

\section{Animal studies}

In vivo tissue (eye) penetration tests showed that Nanosuspension $B$ had the highest Cyc-A concentration in aqueous humor comparing other formulations (Table 3) Also, it was found that Nanosuspension $B$ had the best Cyc-A release profile (Figure 3 and 4). 
There was no adverse effect found after application of Depores and Restasis. However, eye irritations were observed during initial experiments using Nanosuspension A application so ocular irritation test was performed just by using Nanosuspension B. Nanosuspension $B$ formulation was applied to the rabbits and their eyes were checked for two weeks. A drop of Nanosuspension B was applied to both eyes of rabbits for 12 hours for 14 days. There were no swelling, discharge, and redness of the conjunctiva observed during the experiment and also at the end of 14 days (Figure 5).
A is generally administered one to two drops a day to the eyes for a period of six months. Cyc-A has also other labeled indications such as vernal keratoconjunctivitis and atopic keratoconjunctivitis in which Cyc-A doses should be higher. The vernal keratoconjunctivitis is a severe and chronic disease which can damage the corneal tissue and cause the loss of vision. Atopic keratoconjunctivitis is a rare but potentially serious condition that will also damage the corneal tissue and cause the loss of vision.

There are some other indications in which Cyc-A is used as off label for preventing patients from graft

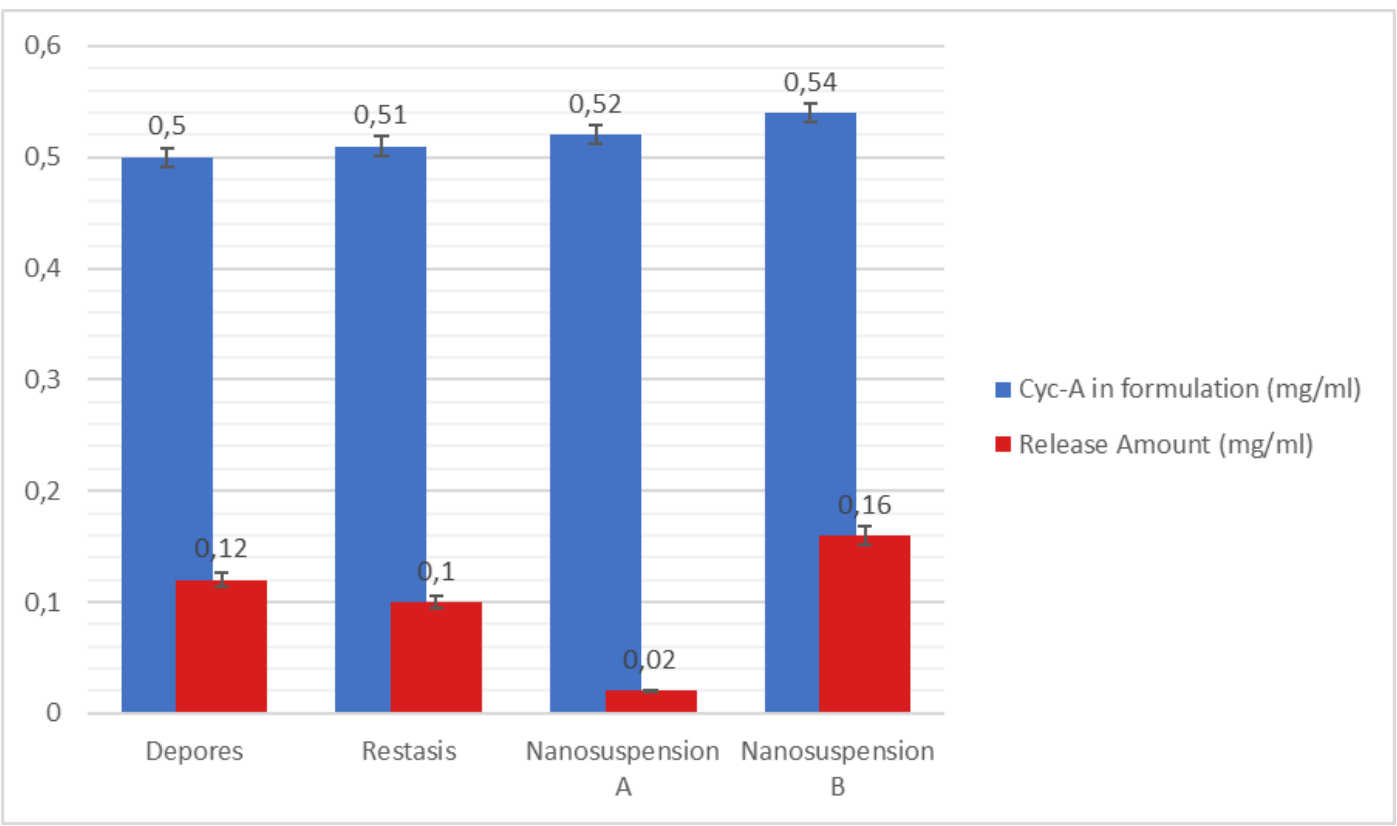

Figure 3. Comparison of Cyc-A concentrations in aqueous humor (red) and formulations (blue) including Depores, Restasis, Nanosuspension $A$, and $B$ (Error bars represent mean $\pm S E M, n=5$ ).

\section{DISCUSSION}

The global market for artificial tears is projected to reach US $\$ 2.0$ billion by 2024 , driven by the rising incidence of DES which is over the years has become one of the major public health concerns. DES is rising in public with the increase in the use of digital screens such as tablets, cell phones, computer screens, etc. which causes prolonged exposure to the blue light. Also, an increase in refractive and cataract surgeries, global climate changes, and the prolonged lifetime in humankind rise the incidence of DES.

Cyc-A is an alternative treatment to artificial tears as it is an immunomodulatory agent that can cure DES or at least lower the daily usage of artificial tears. Ophthalmologists have used Cyc-A not only in keratoconjunctivitis sicca caused by the Sjögren syndrome but also in mild to severe DES cases. Cyc- versus host disease (GVHD) after corneal transplantation, for the treatment of corneal haze which is a complication of the laser refractive surgery, for the treatment of acute conjunctivitis in StevensJohnson Syndrome, and finally for the treatment of Adenoviral keratoconjunctivitis.

GVHD is still one of the major complications of corneal transplantation surgery. To prevent GVHD, long term topical steroids are prescribed to the patients. Not proven yet but some clinicians also add Cyc-A to the therapy for the additive immunomodulatory effect. Photorefractive keratectomy and its variants are refractive surgery technics, in which laser beams are applied to the anterior stroma just below the bowman layer and in some cases, corneal haze can be seen for three months after the surgery. Corneal haze is believed to 
be caused by multipotent stem cells which are activated in the stroma by the laser beams or by the migration of the ones from the blood circulation to the stromal tissue. The corneal haze is a challenging complication of refractive surgery. For the treatment, prolonged use of topical steroids and artificial tears and high dose of Vitamin-C are preferred. Some ophthalmologists add Cyc-A to the treatment. with BAK caused lower Cyc-A concentration in aqueous humor comparing Nanosuspension B. It was evaluated that BAK which was used for reducing the surface tension of the formulation might trigger decrease in nasolacrimal drainage. BAK as a surfaceactive agent has also antiseptic effect but it was observed that nanosuspension A application caused eye irritation in our study. Although there were studies

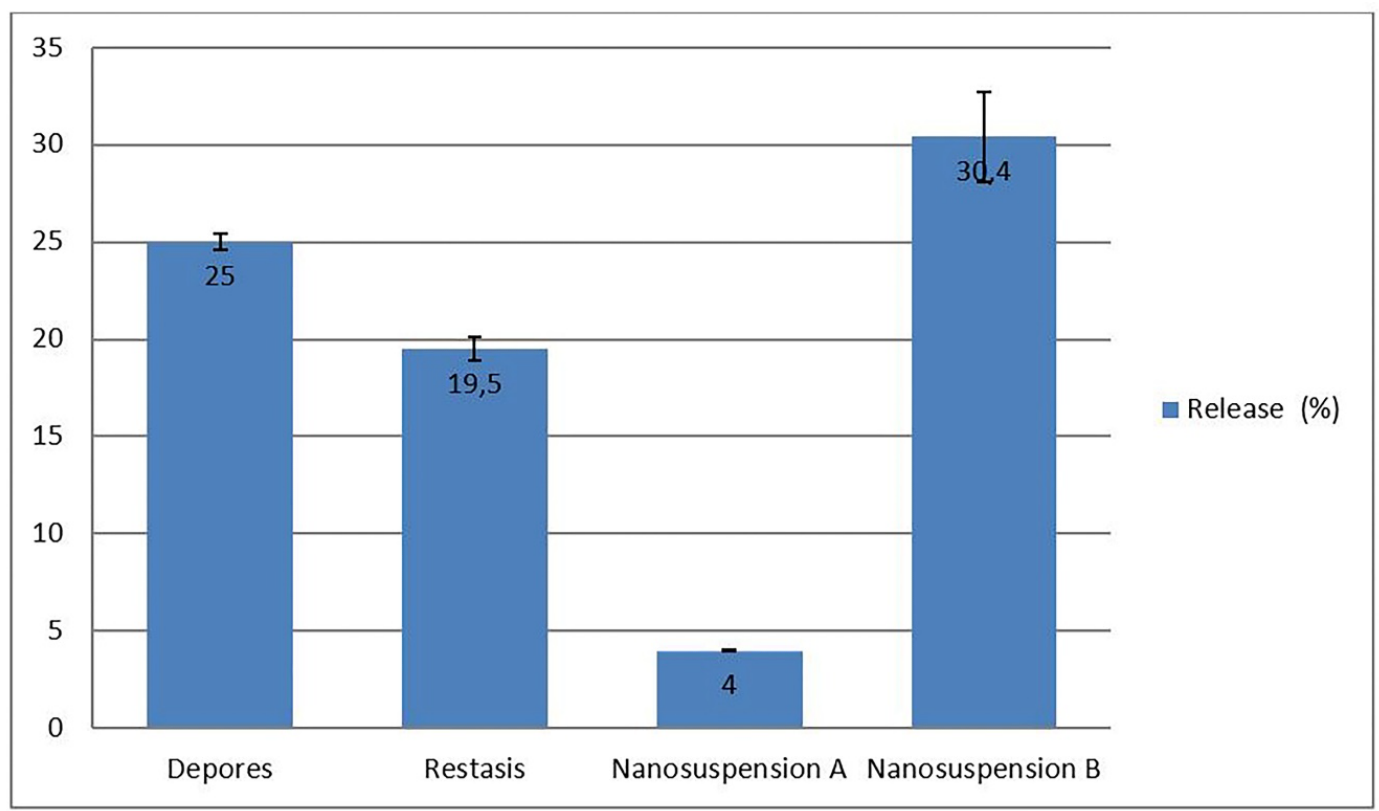

Figure 4. The in vivo test results of Cyc-A release for Depores, Restasis, Nanosuspension A, and B (Error bars represent mean \pm SEM, $n=5$ ).

Stevens-Johnson Syndrome is a self-limiting disease so that controlling the acute phase is very important. Topical steroids and Cyc-A micro emulsion can control acute conjunctivitis. Adenoviral keratoconjunctivitis is also a challenging disease for the ophthalmologists as the virus is very potent and sad but there is no treatment available. However, the use of topical steroids is controversial and the treatment depends on the immunity of the patients. Some ophthalmologists use Cyc-A with topical steroids for the treatment of the subepithelial opacities in stage 2 adenoviral keratoconjunctivitis and anterior stromal opacities in stage 3 adenoviral keratoconjunctivitis but delivering Cyc-A to the target tissues is always a problem for the eye because of the corneal and conjunctival epithelial barriers which prevent Cyc-A to pass through the underlying tissue compartments.

In our study, two different nanosuspension formulations of Cyc-A were prepared as nanosuspension $A$ and $B$. It was observed that Nanosuspension A application which was prepared which used same amount of surface-active agent in the literature, our study was the one which observed eye irritation. It was concluded that eye irritation was the negative effect of the surface-active agent based nanosuspensions which were prepared without nonsteroidal anti-inflammatory ingredients which could reduce the inflammation (24).

Therefore, we prepared nanosuspension $B$ without using BAK. We performed ocular irritation test for 14 days by using 2 rabbits. We observed the potential presence of eye symptoms including swelling, discharge, and redness of the conjunctiva for 14 days but none of the subjects exhibited any irritation after nanosuspension B applications.

The characterization of formulations such as the particle size, polydispersity index, EE, and zeta potential is also an important feature that regulates the saturation solubility, dissolution rate, physical stability, and the biological performance. Particle sizes of formulations were found to be similar in the study. The polydispersity index is a measure of the distribution of molecular mass in a given polymer 
sample (25). The polydispersity index of a nanosuspension should be as low as possible for long-term stability (26). All formulations that we used in the study had similar polydispersity indexes ranging from 25 to $37 \%$, therefore, it was concluded that all of them were comparable. It was also concluded that particle distribution and polydispersity indexes of all formulations in these ranges were not have any negative effect.

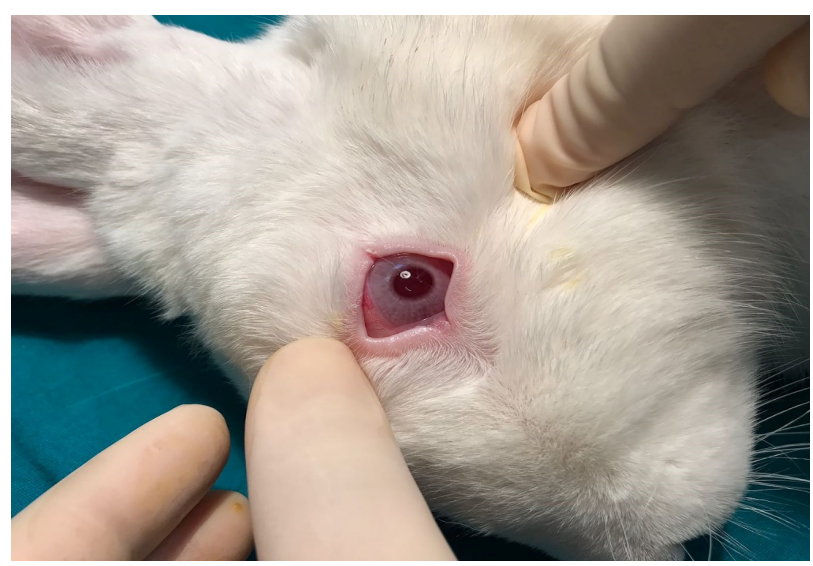

Figure 5. The physical appearance of the rabbit eye after 14 days of Nanosuspension B application.

It was noticed that nanosuspension $B$ had a positive value of $27,5 \mathrm{mV}$. This finding is critical as it is well known that biological membranes including eye tissue membranes have a negative surface potential. Membrane potential (also known as transmembrane potential or membrane voltage) is the difference in electric potential between the interior and the exterior of a biological cell. With respect to the exterior of the cell, typical values of membrane potential are ranging from $-40 \mathrm{mV}$ to $-80 \mathrm{mV}$ (25). Therefore, a particle having a positive surface potential is more likely to interact with tissue cell membranes and it may find a better opportunity to stay at the surface and to release the drug for a longer period. It can be also penetrating through the cell membranes with higher amounts (27). This might be the exact reason why we obtained a better value with nanosuspension $B$.

Although lower values were obtained when BAK was present in the formulations including Depores, Restasis, and nanosuspension $A$, the encapsulation efficiency of nanosuspension $B$ was found to be higher than $95 \%$. It was concluded that preparation of a formulation without a surface active agent like BAK resulted in significant enclosure of Cyc-A into the nanoparticles.

Finally, there were no adverse effects observed after the application of Depores, Restasis, and nanosuspension B like studies which performed intravenous administration of Cyc-A nanosuspensions to dogs and rabbits $(28,29)$.

In this study, two types of Cyc-A containing nanosuspensions were prepared by the quasiemulsion solvent evaporation technique, which is an easy, inexpensive, and readily scalable method to prepare nanoparticles. Then, we investigated the potential of Cyc-A loaded Eudragit $\mathrm{S} 100$ based nanosuspension formulations for ocular delivery of Cyc-A by comparing them two marketed ophthalmic emulsion including Depores and Restasis.

In vitro Cyc-A release profile tests showed that CycA loaded Eudragit $\mathrm{S} 100$ based nanosuspension formulation without BAK had higher Cyc-A release in mean of percentage comparing two marketed ophthalmic Cyc-A emulsions which had similar particle size and polydispersity index. However, nanosuspension $B$ had a positive zeta potential which could be the reason of higher Cyc-A release. Nanosuspension B was also found as the highest Cyc-A concentration in aqueous humor with a good ocular tolerability profile.

The nanosuspensions have ideal mean particle size range for ophthalmic applications with a positive surface charge. Besides, they enable corneal adhesion and they have good stability upon storage. Therefore, it is concluded that Cyc-A loaded Eudragit $S 100$ based nanosuspension which contains $C y c-A$ is an ideal candidate for the treatment of the dry eye.

\section{CONCLUSION}

In this study, two type of Cyc-A containing nanosuspensions were prepared by the quasiemulsion solvent evaporation technique which is an easy, inexpensive, and readily scalable method to prepare nanoparticles. Then, we investigated the potential of Cyc-A loaded Eudragit $\mathrm{S} 100$ based nanosuspension formulations for ocular delivery of Cyc-A by comparing them two marketed ophthalmic emulsion including Depores and Restasis.

In vitro Cyc-A release profile tests showed that CycA loaded Eudragit $\mathrm{S} 100$ based nanosuspension formulation without BAK had higher Cyc-A release in mean of percentage comparing two marketed ophthalmic Cyc-A emulsions which had similar particle size and polydispersity index. However, nanosuspension $B$ had a positive zeta potential which could be the reason of higher Cyc-A release. Nanosuspension B was also found as the highest 
Cyc-A concentration in aqueous humor with a good ocular tolerability profile.

The nanosuspensions have ideal mean particle size range for ophthalmic applications with a positive surface charge. Besides, they enable corneal adhesion and they have good stability upon storage. Therefore, it is concluded that Cyc-A loaded Eudragit $\mathrm{S} 100$ based nanosuspension which contains Cyc-A is an ideal candidate for the treatment of the dry eye.

Ethical approval: This study was approved by Gazi University Local Ethics Committee for Animal Experiments (G.U.ET-17.012) Conflict of Interest: The authors report no conflict of interest. Peer-review: Externally peer-reviewed.

\section{REFERENCES}

1. Pflugfelder SC, Solomon A, Stern ME. The Diagnosis and Management of Dry Eye. Cornea 2000;19: 644-649.

2. Pflugfelder SC, Paiva CS, Villarreal AL, Stern ME. Effects of Sequential Artificial Tear and Cyclosporine Emulsion Therapy on Conjunctival Goblet Cell Density and Transforming Growth Factor-b2 Production. Cornea 2008;27: 64-69.

3. Perry HD, Donnenfeld ED.Dry eye diagnosis and management in 2004. Current Opinion in Ophthalmology 2004;15: 299-304.

4. Small DS, Acheampong A, Reis B, Stern K, Stewart W, Berdy $G$ et al. Blood Concentrations of Cyclosporin A During Long-Term Treatment With Cyclosporin A Ophthalmic Emulsions in Patients With Moderate to Severe Dry Eye Disease. Journal of Ocular Pharmacology and Therapeutics 2002;18: 411-418.

5. Wilson SE, Stulting RD. Agreement of Physician Treatment Practices With the International Task Force Guidelines for Diagnosis and Treatment of Dry Eye Disease. Cornea 2007;26: 284-289.

6. Stevenson D, Tauber J, Reis B. Efficacy and safety of cyclosporin A ophthalmic emulsion in the treatment of moderate to severe dry eye desease. Ophthalmology 2000;107: 967-974.

7. Moon WJ, Lee HJ, Shin KC, Wee WR, Lee JH, Kim MK. Short term effects of topical cyclosporine and viscoelastic on the ocular surface in patients with dry eye. Korean Journal of Ophthalmology 2007;21: 189-194.

8. Wilson SE, Perry HD. Long term resolution of chronic dry eye symptoms and signs after topical cyclosporine treatment. Ophthalmology 2007;114: 76-79.
9. Stern ME, Gao J, Schwalb TA, Ngo M, Tieu DD, Chan CC et al Conjunctival T-Cell Subpopulations in Sjogren's and Non-Sjogren's Patients with Dry Eye. IOVS 2002;43: 2609-2614

10. Foulks GN. Pharmacological Management of Dry Eye in the Elderly Patient. Drugs Aging 2008;25: 105-118.

11. Pflugfelder SC. Antiinflammatory Therapy for Dry Eye. American Journal of Ophthalmology 2004; 137: 337-342.

12. Perry HD, Donnenfeld ED. Topical $0.05 \%$ cyclosporin in the treatment of dry eye. Expert Opinion 2004: 2099-2107.

13. Sall KN, Cohen SM, Christensen MT, Stein JM. An Evaluation of the Efficacy of a CyclosporineBased Dry Eye Therapy When Used With Marketed Artificial Tears as Supportive Therapy in Dry Eye. Eye \& Contact Lens 2006;32: 21-26.

14. Kunert KS, Tisdale AS, Gipson IK. Goblet Cell Numbers and Epithelial Proliferation in the Conjunctiva of Patients With Dry Eye Syndrome Treated With Cyclosporine. Arch Ophthalmol 2002;120: 330-337.

15. Foulks GN. Topical Cyclosporine for Treatment of Ocular Surface Disease: 105-122

16. Turner K, Pflugfelder SC, Ji Z,Feuer WF, Stern $\mathrm{M}$, Reis BL. Interleukin- 6 Levels in the Conjunctival Epithelium of Patients with Dry Eye Disease Treated with Cyclosporine Ophthalmic Emulsion. Cornea 2000;19: 492-496

17. Acheampong AA, Shackleton M, Tang-Liu DD, et al. Distribution of cyclosporin A in ocular tissues after topical administration to albino rabbits and beagle dogs. Curr Eye Res. 1999;18:91-103.

18. R. H. Muller, C. Jacobs, and O. Kayser. Nanosuspensions as particulate drug formulations in therapy. $\mathrm{P}-557$ the future. Adv Drug Deliv Rev. 47(1):3-19 (2001).

19. K. Kreuter. Peroral administration of nanoparticles. Advanced Drug Delivery Reviews. 7:71-86 (1991).

20. Liversidge GG, Cundy KC.Particle size reduction for improvement of oral bioavailability of hydrophobic drugs: Absolute oralbioavailability of nanocrystalline danazol in beagle dogs.Int $\mathrm{J}$ Pharm. 1995; 125(1): 91-97.

21. Kawashima, Y., Niwa, T., Handa, T., Takeuchi, H., Iwamoto, T., Itoh, K., 1989. Preparation of controlled-release microspheres of ibuprofen with acrylic polymers by a novel quasi-emulsion 
solvent diffusion method. J. Pharm. Sci. 78, 6872.

22. Pignatello, R.,Vandelli, M.A., Giunchedi, P., Puglisi, G., 1997. Properties of tolmetin-loaded Eudragit RL100 and RS100 microparticles prepared antiinflamby different techniques. S.T.P. Pharma Sci. 7, 148-157.

23. Tveden-Nyborg $P$, Bergmann TK, Jessen $N$, Simonsen U, Lykkesfeldt J. BCPT policy for experimental and clinical studies. Basic Clin Pharmacol Toxicol. 2021 Jan;128(1):4-8.

24. Swamy N.G.N., Zaheer Abbas2 and Santosh Kumar I.H., Eudragit RS 100 nanosuspensions for the controlled ophthalmic delivery of diclofenac sodium, Thai J. Pharm. Sci. 2013: 37: 157-170.

25. Nanocomposıx's Guıde To Dynamıc Light Scatterıng Measurement And Analysıs. February 2015, V 1.4

26. A. Amrite, H. Edelhauser, S. Singh, and U. Kompella. Effect of circulation on the disposition and ocular tissue distribution of $20 \mathrm{~nm}$ nanoparticles after periocular administration, Mol. Vis. 2008: 14; 150-160.

27. S. Das, and P.K. Suresh. Nanosuspension: a new vehicle for the improvement of the delivery of drugs to ocular surface. Application to amphotericin B, Nanomedicine 2011: 7: 242-247.

28. Mahendra Nakaranı, Priyal Patel, Jayvadan Patel, Pankaj Patel, Rayasa S. R. Murthy, Subhash S. Vaghanı. Cyclosporine ANanosuspension: Formulation, Characterization and In Vivo Comparison with a Marketed Formulatio. Sci Pharm. 2010; 78: 345-361 doi:10.3797/scipharm.0908-1

29. Rowe RC, Sheskey PJ, Owen SC. Handbook of Pharmaceutical Excipients. 5th ed. London: PhP. 2006: 449-453, 535-538. 